\title{
Biochemical alterations during medication withdrawal in Parkinson's disease with and without neuroleptic malignant-like syndrome
}

\author{
M Ueda, M Hamamoto, H Nagayama, S Okubo, S Amemiya, Y Katayama
}

\begin{abstract}
The object was to assess alterations in CSF concentrations of monoamine metabolites during withdrawal of medication in patients with Parkinson's disease in relation to the presence or absence of episodes resembling neuroleptic malignant syndrome (NMS). This syndrome is a fatal condition developing after neuroleptic therapy, and a neuroleptic malignant-like syndrome (NMLS) may also occur after withdrawal of antiparkinsonian drugs in patients with Parkinson's disease. Previous biochemical assays showed that the CSF concentration of the dopamine metabolite homovanillic acid (HVA) is an independent prognostic factor for development of NMLS in patients with Parkinson's disease. In the present study, CSF concentrations of HVA, the noradrenaline (norepinephrine) metabolite 3-methoxy4-hydroxyphenylethylene glycol, and the serotonin metabolite 5-hydroxyindole acetic acid were assayed using high performance liquid chromatography with electrochemical detection. The study population consisted of nine patients with Parkinson's disease with NMLS and 12 without NMLS, in whom metabolites were assayed during both withdrawal and remedicated periods. Concentrations of HVA in the CSF were significantly lower during the withdrawal period than the medicated period regardless of whether patients developed NMLS, and HVA concentrations were comparably increased after remedication in both groups. However, HVA concentrations were significantly lower in patients with NMLS than in those without NMLS during both withdrawal and medicated periods. Other metabolites showed no significant differences. The present data provide further biochemical evidence for extremely suppressed central dopaminergic activity during NMLS, which may indicate a narrow safety margin for medication withdrawal in patients with Parkinson's disease.

(f Neurol Neurosurg Psychiatry 2001;71:111-113)
\end{abstract}

Keywords: neuroleptic malignant-like syndrome; Parkinson's disease; homovanillic acid; 3-methoxy-4hydroxyphenylethylene glycol; 5-hydroxyindole acetic acid

Neuroleptic malignant syndrome (NMS) was first described by Delay and Deniker in 1968 as a fatal disorder developing after neuroleptic therapy. ${ }^{1}$ An NMS-like state (neuroleptic malignant-like syndrome; NMLS) may also occur after withdrawal of antiparkinsonian drugs in patients with Parkinson's disease. ${ }^{23}$ We have previously reported that patients with clinically advanced Parkinson's disease are at high risk for NMLS, and that CSF concentration of the dopamine metabolite homovanillic acid (HVA) is an independent prognostic factor for development of NMLS in patients with Parkinson's disease. ${ }^{4}$ Thus central monoamine metabolism, especially dopamine metabolism, may be involved in the pathogenesis of NMLS. Although monoamine metabolite concentrations in the CSF in patients with NMS have been studied, most of the investigations involved only single cases of psychiatric patients after neuroleptic treatment. ${ }^{5-7}$

The present study sought to determine biochemical alterations during medication withdrawal in patients with Parkinson's disease in relation to the presence or absence of NMLS, measuring monoamine metabolite concentrations in CSF as indicators of central biogenic amine transmitter metabolism.

\section{Materials and methods}

During a 5 year study period (1994-9), 29 patients with Parkinson's disease were admitted to our service because of worsened extrapyramidal symptoms after medication withdrawal. Fourteen patients developed NMLS whereas the remaining 15 patients did not. Nine patients with NMLS were included in an earlier prospective study, ${ }^{4}$ and five additional patients with NMLS were subsequently admitted to our service because of their NMS-like episodes. Of the 29 patients, eight underwent CSF examination only at a single point and were excluded, leaving 21 patients who received examinations both at admission and after remedication with antiparkinsonian drugs. There were 13 men and eight 
women with a mean age of 77.8 years, a mean disease duration of 6.0 years, and a mean Hoehn and Yahr score of 3.7. They discontinued their medications because of their refusal to take drugs, probably due to adverse effects of psychosis by antiparkinsonian medications or anorexia. All patients successfully recovered from their NMLS or worsened bradykinetic states on restarting the withdrawn drugs. The clinical diagnosis of Parkinson's disease was based on the presence of two or more cardinal symptoms including tremor, rigidity, akinesia, and postural instability, ${ }^{8}$ and responsiveness to levodopa. As in our previous study, ${ }^{4}$ the clinical diagnosis of NMLS was based on the presence of two or more cardinal manifestations, including hyperpyrexia, altered consciousness, severe extrapyramidal symptoms, autonomic instability, and increased serum creatine kinase concentrations. The criteria of Pope et al were used to set thresholds for each of these manifestations in the present study, except that more than $185 \mathrm{IU} / 1$ was considered to define increased serum creatine kinase concentration according to the upper normal limit of our previous study. ${ }^{4}$

Lumbar CSF was obtained immediately after admission (withdrawal period) and after an overnight fast after recovery under unchanged daily dosages of antiparkinsonian medications for at least 1 month before CSF sampling (medicated period). Informed consent was obtained from each patient or from a relative (if the patient exhibited altered consciousness). The first 3-4 ml CSF were used for routine examinations and the next $6-7 \mathrm{ml}$ were used to measure monoamine metabolite levels. The CSF samples were immediately frozen and stored at $-70^{\circ} \mathrm{C}$ until analysis. In the medicated period, the medications at CSF examination were levodopa in combination with carbidopa (levodopa:carbidopa $=10: 1$ ) in

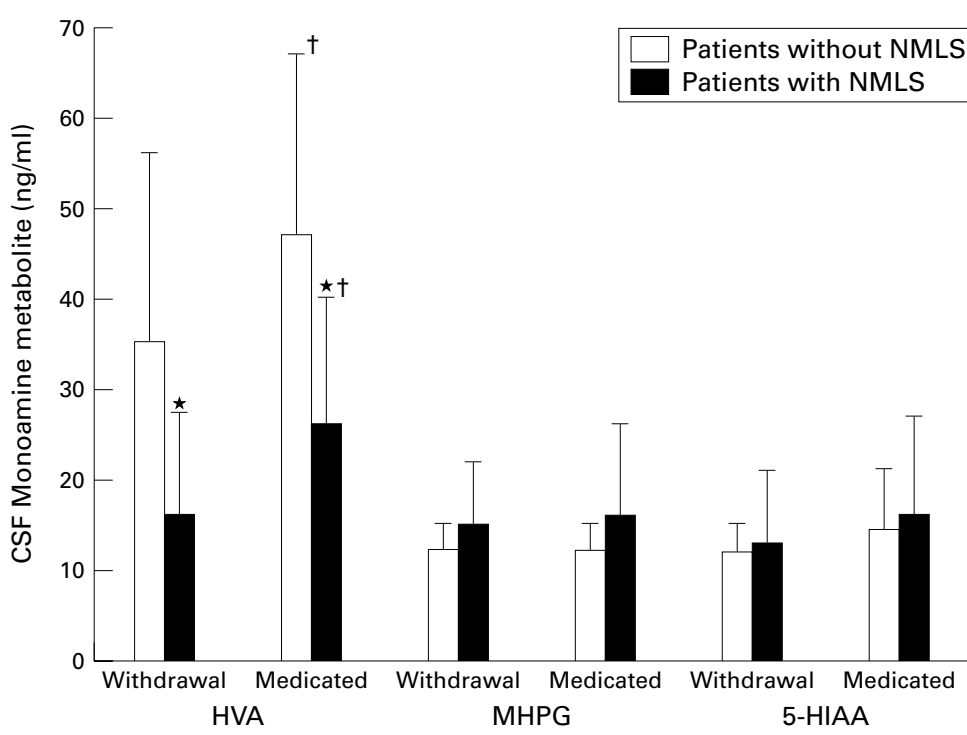

Figure 1 Monoamine metabolite concentrations in CSF during medicated and withdrawal periods in parkinsonian patients with and without NMLS. Patients experiencing an NMLS had significantly lower HVA concentrations than those who did not during both withdrawal and remedicated intervals ( ${ }^{*} p<0.05$ v non-NMLS group, Scheffe's $F$ test). The lower HVA concentrations found during drug withdrawal increased significantly after restarting medication in both patient groups ( $t p<0.05 v$ withdrawal interval, paired t test).
21 patients (mean daily dosage, $214 \mathrm{mg}$ levodopa; range, 100-600 mg), dopamine agonists in 14 patients $(9.5 \mathrm{mg} ; 1.5-27.5 \mathrm{mg})$, and droxidopa ${ }^{10}$ a synthetic precursor of noradrenaline (norepinephrine), in eight patients (675 $\mathrm{mg}$; 300-900 mg). The daily dosage of dopamine agonist in each patient was transformed into mg bromocriptine equivalents as in our previous study ${ }^{4}$ in accordance with Calne's suggestion ${ }^{11}$ that $1 \mathrm{mg}$ pergolide is clinically equivalent to $10 \mathrm{mg}$ bromocriptine. Assays of HVA, the noradrenaline metabolite 3-methoxy-4-hydroxyphenylethylene glycol (MHPG), and the serotonin metabolite 5-hydroxyindole acetic acid (5-HIAA) were carried out by high performance liquid chromatography with electrochemical detection, as described previously. ${ }^{4}$

Analysis of variance (ANOVA) with Scheffe's $F$ test was used for comparison of age, disease duration, Hoehn and Yahr score, daily dosages of antiparkinsonian drugs, monoamine metabolite concentrations in CSF, and changes in metabolite concentrations between withdrawal and medicated periods in NMLS and non-NMLS groups. A paired $t$ test was also performed to compare changes in monoamine metabolite concentrations in CSF from the withdrawal to the medicated periods in the NMLS and the non-NMLS groups. Data are presented as means (SD), and statistical significance was considered as $\mathrm{p}<0.05$.

\section{Results}

There were no significant differences between the NMLS and the non-NMLS groups in age (mean 75.3 v 79.6 years), disease severity assessed by Hoehn and Yahr score (4.0 v 3.4), or disease duration (5.3 $v 6.4$ years). Levodopa/ carbidopa was given in nine patients (mean daily dosage $233 \mathrm{mg}$ levodopa) in the NMLS group, and in 12 patients (200 mg levodopa) in the non-NMLS group. Dopamine agonists and droxidopa were given in five $(9.5 \mathrm{mg})$ and three (700 mg) patients in the NMLS group, and in nine $(9.5 \mathrm{mg})$ and five $(660 \mathrm{mg})$ patients, in the non-NMLS group, respectively. There were also no significant differences in the daily dosages of antiparkinsonian medications between the groups.

Mean CSF concentrations of HVA, MHPG, and 5-HIAA during medication withdrawal in the NMLS and the non-NMLS groups were 16.0 ข $34.6(\mathrm{p}=0.027), 14.9$ $12.3(\mathrm{p}=0.281)$, and $12.6 v 12.4(\mathrm{p}=0.918) \mathrm{ng} / \mathrm{ml}$, respectively. In the medicated period, these concentrations were 26.3 v $46.9(\mathrm{p}=0.014), 16.3$ v 12.2 $(\mathrm{p}=0.186)$, and $15.6 \vee 14.3(\mathrm{p}=0.737) \mathrm{ng} / \mathrm{ml}$, respectively. Only the HVA concentrations were significantly lower in the NMLS group than in the non-NMLS group, and this was the case during both withdrawal and medicated periods (fig 1). Concentrations of HVA during the medicated period in the NMLS group were not statistically different from those during the withdrawal period in the non-NMLS group $(p=0.318)$. In the medicated period, the concentrations of HVA in CSF were not different between patients treated with and without dopamine agonist $(p=0.543)$, and the MHPG 
concentrations in CSF showed no differences between patients treated with and without droxidopa $(\mathrm{p}=0.141)$.

Paired $t$ tests showed that concentrations of HVA in CSF were significantly lower in the withdrawal period than in the medicated period both in the NMLS and non-NMLS groups $(p=0.018$ and 0.001$)$, whereas no differences in concentrations were found for MHPG $(p=0.631$ and 0.864$)$ or 5-HIAA $(\mathrm{p}=0.361$ and 0.350$)$. The increase in the CSF concentrations of HVA between withdrawal and medicated periods did not differ between the groups $(p=0.647)$.

\section{Discussion}

The present study demonstrated that HVA concentrations in CSF were significantly decreased during the withdrawal period compared with the medicated period, regardless of whether patients developed an NMLS. In addition, the increase in HVA concentrations following remedication were similar in the NMLS and the non-NMLS groups. Durso et al showed that about half of the HVA in the CSF turns over within 6 hours, and suggested that the HVA pool in CSF is remarkably dynamic in patients with Parkinson's disease. ${ }^{12}$ Not only levodopa but also dopamine agonists potentially influence the HVA concentration in CSF, although the HVA concentrations were not different between patients treated with and without dopamine agonists in the present Parkinson's disease series with all patients taking levodopa. Therefore, abrupt medication withdrawal, especially that of levodopa, probably induced a sudden decrease in HVA concentration in CSF in the present patients with Parkinson's disease. However, not all patients with Parkinson's disease who discontinue their medications develop an NMLS, suggesting that additional factors contribute to NMLS development. The present data showed that the HVA concentrations were significantly lower in the NMLS group than in the non-NMLS group both in the withdrawal and the medicated phase, and that the HVA concentrations during the medicated period in the NMLS group were similar to those during the withdrawal period in the non-NMLS group. This is in agreement with our previous study, ${ }^{4}$ in which we estimated that the relative risk for NMLS increased about 3-fold for each 10 $\mathrm{ng} / \mathrm{ml}$ decrease in HVA concentration in CSF in patients with Parkinson's disease. Lower baseline HVA concentrations seem to indicate a narrow safety margin during drug withdrawal, leading to NMLS susceptibility. Furthermore, readministration of the withdrawn drugs improved clinical and biochemical abnormalities in the present patients with NMLS, confirming the efficacy of dopaminergic agents in reversing the NMLS in Parkinson's disease.
Concentrations of CSF MHPG, and 5-HIAA remained unchanged during remedication after withdrawal both in the NMLS and the nonNMLS groups, conflicting with several previous findings. The MHPG concentrations in CSF were reportedly increased during acute phase NMS, suggesting probable noradrenergic hyperactivity. ${ }^{7}$ Changes found in 5-HIAA concentrations in CSF during acute phase NMS are inconsistent, with both increases and decreases reported. ${ }^{67}$ However, these findings were in psychiatric patients with neuroleptic treatment, not in Parkinson's disease with medication withdrawal. Because not only dopaminergic but also noradrenergic and serotonergic system degenerations are involved in Parkinson's disease pathology, ${ }^{13}$ NMLS occurring in the present Parkinson's disease series might show neither noradrenergic nor serotonergic hyperactivity.

In conclusion, we present biochemical evidence for extremely suppressed central dopaminergic activity during NMLS, defining a narrow safety margin for medication withdrawal in a subpopulation of patients with Parkinson's disease. The present data strongly suggest that central dopaminergic suppression contributes to NMLS pathogenesis in the Parkinson's disease population.

We thank Professors Thaddeus S Nowak Jr and Ronald F Pfeiffer from the Department of Neurology, University of Tennessee, Health Science Center, for their helpful comments on this manuscript.

1 Delay J, Deniker P. Drug-induced extrapyramidal syndromes. In: Vinken PJ, Bruyn GW, eds. Handbook of clinical neurology; diseases of the basal ganglia. Amsterdam: North Holland, 1968:248-66.

2 Henderson VW, Wooten GF. Neuroleptic malignant syndrome: a pathogenetic role for dopamine receptor blockade? Neurology 1981;31:132-7.

3 Toru M, Matsuda O, Makiguchi K, et al. Neuroleptic malignant syndrome-like state following a withdrawal of antiparkinsonian drugs. F Nerv Ment Dis 1981;169:324-7.

4 Ueda M, Hamamoto M, Nagayama $\mathrm{H}$, et al. Susceptibility to neuroleptic malignant syndrome in Parkinson's disease. Neurology 1999;52:777-81.

5 Granato JE, Stern BJ, Ringel A, et al. Neuroleptic malignant syndrome: successful treatment with dantrolene and bromocriptine. Ann Neurol 1983;14:89-90.

6 Tollefson GD, Garvey MJ. The neuroleptic syndrome and central dopamine metabolites. F Clin Psychopharmacol $1984 ; 4: 150-3$

7 Nishijima K, Ishiguro T. Neuroleptic malignant syndrome: a study of CSF monoamine metabolism. Biol Psychiatry $1990 ; 27: 280-8$

8 Calne DB, Snow BJ, Lee C. Criteria for diagnosing Parkinson's disease. Ann Neurol 1992;32(suppl):125-7.

9 Pope HG Jr, Keck PE Jr, McElroy SL. Frequency and presentation of neuroleptic malignant syndrome in a large psychiatric hospital. Am f Psychiatry 1986;143:1227-33.

10 Suzuki T, Sakodo S, Ueji M, et al. Treatment of parkinsonism with L-threo-3,4-dihydroxyphenylserine: a pharmacokinetic study. Neurology 1984;34:1446-50.

11 Calne DB. Treatment of Parkinson's disease. $N$ Engl f Med 1993;329:1021-7.

12 Durso R, Evans JE, Josephs E, et al. Central levodopa metabolism in Parkinson's disease after administration of stable isotope-labeled levodopa. Ann Neurol 1997;42:3004.

13 Halliday GM, Li YW, Blumbergs PC, et al. Neuropathology of immunohistochemically identified brainstem neurons in Parkinson's disease. Ann Neurol 1990;27:373-85. 